\title{
Wigner's infinite spin representations and inert matter
}

\author{
Bert Schroer $^{2,1, \mathrm{a}}$ \\ ${ }^{1}$ Present address: CBPF, Rua Dr. Xavier Sigaud 150, Rio de Janeiro 22290-180, Brazil \\ ${ }^{2}$ Institut für Theoretische Physik FU-Berlin, Arnimallee 14, 14195 Berlin, Germany
}

Received: 10 May 2016 / Accepted: 9 May 2017 / Published online: 31 May 2017

(C) The Author(s) 2017. This article is an open access publication

\begin{abstract}
Positive energy ray representations of the Poincaré group are naturally subdivided into three classes according to their mass and spin content: $m>0, m=0$ finite helicity and $m=0$ infinite spin. For a long time the localization properties of the massless infinite spin class remained unknown, until it became clear that such matter does not permit compact spacetime localization and its generating covariant fields are localized on semi-infinite spacelike strings. Using a new perturbation theory for higher spin fields we present arguments which support the idea that infinite spin matter cannot interact with normal matter and we formulate conditions under which this also could happen for finite spin $s>1$ fields. This raises the question of a possible connection between inert matter and dark matter.
\end{abstract}

\section{Wigner's infinite spin representation and string-localization}

Wigner's famous 1939 theory of unitary representations of the Poincare group $\mathcal{P}$ was the first systematic and successful attempt to classify relativistic particles according to the intrinsic principles of relativistic quantum theory [1]. As we know nowadays, his massive and massless spin/helicity class of positive energy ray representations of $\mathcal{P}$ does not only cover all known particles, but their "covariantization" [2] leads also to a complete description of all covariant pointlocal (pl) free fields. For each covariant Lorentz transformation property compatible with physical spin or helicity there exists a pl field.

It is not necessary that these free fields permit a characterization as Euler-Lagrange fields; in fact the attempt to describe higher spin fields in this way and pass to quantum fields by canonical quantization or use other ways to

Dedicated to the memory of Robert Schrader.

a e-mail: schroer@zedat.fu-berlin.de start from a classical field creates problems of their own. On the other hand the construction of free fields starting from Wigner's representation theory does not require one to rely on any "quantization parallelism" to classical structures.

The idea that a more fundamental theory should not rely on structural properties of a less basic one can also be upheld in the presence of interactions. Defining a first order Lorentz invariant interaction densities in terms of products of free fields and using the Epstein-Glaser [3] formulation of renormalization theory permits a perturbative cutoff- and regularization-free higher order inductive implementation of the causal localization principle in a positivity-obeying Wigner-Fock Hilbert space without a reference to the classical field formalism. Renormalizable theories in this setting are those for which this higher order induction increases the model-defining first order parameters by an at most finite number of "counter term" parameters.

When higher spin $s \geq 1$ fields participate in the interaction there are no positivity-obeying renormalizable couplings. The traditional way out has been to skip positivity in the calculations and save it by restricting the result. A total abandonment would have been a disaster because quantum theory would lose its important probability interpretation. Quantum gauge theory is the result of a compromise; to keep renormalizability of $s=1$ interactions one accepts that part of the formalism becomes unphysical; this includes all fields which carry electric charges.

Quantum gauge theory keeps some formal relation with classical gauge theory; but whereas in the classical case gauge symmetry is a bona fide local ${ }^{1}$ symmetry which is in agreement with the conceptual structure of classical field theory, the clash with the pivotal positivity

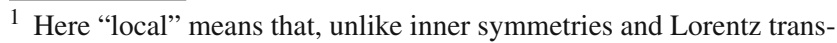
formations which act everywhere in spacetime, gauge transformation may act in some compact spacetime region and leave the outside unchanged.
} 
property of quantum theory suggests that quantum gauge symmetry is not a physical symmetry within a quantum field theory but rather a formal device which extracts a positivity-obeying physical subtheory from an unphysical formalism.

Hence it is not surprising that the canonical quantization of classical gauge theory causes additional problems on the quantum side. In order to reconcile the classical gauge structure with properties of noncommuting operators one has to pay the high price of trading the Hilbert space with an indefinite metric Krein space and in addition to unphysical negative metric degrees of freedom one also has to add "ghost" degrees of freedom. The resulting perturbative BRST rules can be shown to secure the perturbative positivity of a subtheory but its "ghostly" rules cannot be derived from the physical principles. One has to be content with the fact that they do the job for which they have been invented, namely to provide perturbative rules which secure the unitary of the $S$-matrix.

The recent observation that Wigner's infinite spin representation class admits no compact causal localization [4] and that its tightest localized quantum fields "live" on semi-infinite strings (space-like rays) $S(x, e)=x+\mathbb{R}_{+} e$, $e^{2}=-1$ [5] led to several new fundamental problems concerning the foundational properties of quantum fields. Besides the obvious question concerning possible physical manifestations of this new form of matter one could also ask whether a better understanding of the interplay between positivity and causal localization could favor the use of string-localized (sl) fields in a positivity-maintaining formulation of interactions involving finite higher spin particles.

There is no reason why such interactions should follow the logic imposed by the quantization of classical gauge theory which led to the problems with quantum positivity and also required to add "phantom degrees of freedom" (ghosts) which, after having played their short distance improving role in renormalized perturbation theory, leave no traces in the perturbative $S$-matrix and local observables. Certainly the perturbative rules of quantum gauge theory have been successful; without their use the Standard Model would not have arrived at its present position.

However, gauge theory falls short of providing positivitymaintaining interpolating fields for its particles and hence does not constitute a full QFT. The care for the conceptual health of a theory has always paid its long-term dividends, even if some discoveries were made by pulling up one's sleeves and looking for computational consistency.

Here the more recent discovery of string-localization of Wigner's infinite spin matter comes in. It helped to find "Occam's razor" which permits one to maintain higher spin renormalizability in terms of only physical degrees of freedom and also extends the physical range by providing physi- cal fields for all particles. Interacting string-local (sl) fields ${ }^{2}$ in the form of interpolating fields of particles are not only perfectly natural in QFT, their presence is even required by the principles of QFT.

The only operators which must maintain their compact localization in the presence of interactions are those of the local observables; their fields must remain pl. The intrinsic noncompact string-localization of the free fields associated to Wigner's infinite spin representation which is in perfect agreement with QFTs causal iocalization principles was the catalyst for studying the relation between positivity and causal localization in the presence of interacting $s \geq 1$ higher spin fields. For this purpose, one had to use the more intrinsic relation of positivity with causal localization referred to as "modular" localization relative to which quantum fields and their local equivalence classes acquire the role of "coordinatizations" of causally localized algebras.

In the remainder of this introduction we recollect standard results of Wigner's intrinsic (no quantization parallelism) description of relativistic particles; the conversion of the irreducible positive representation spaces of the Poincaré group into causally localized pl covariant fields and their sl counterparts will be left to the next section.

All positive energy representations are "induced" from irreducible representations of the "little group". This subgroup of the Lorentz group is the stability group of a conveniently chosen reference momentum on the forward mass shell $p_{0}^{2}-\mathbf{p}^{2}=m^{2}$ inside the forward light cone $V_{+}$, respectively, for $m=0$ on its surface $\partial V_{+}$. For $m>0$ this little group is a rotation subgroup of the Lorentz group and for $m=0$ the noncompact Euclidean $E(2)$. Whereas the massive representation class ( $m>0, s=n / 2$ ), of particles with mass $m$ and spin $s$ covers all observational known massive particles (here referred to as the first Wigner class), the massless representations split into two quite different classes.

For the finite helicity representations the $E(2)$ subgroup of Lorentz-"translations" are trivially represented ("degenerate" representations), so that only the abelian rotation subgroup $U(1) \subset E(2)$ remains; this accounts for the semiinteger helicity $\pm|h|,|h|=\frac{n}{2}$ representations (the second Wigner class). The third Wigner class is induced by faithful unitary representations of $E(2)$. Being a noncompact group, they are necessarily infinite dimensional and their irreducible components are characterized in terms of a continuous PauliLubanski invariant $\kappa$.

Since this invariant for massive representations is related to the spin as $\kappa^{2}=m^{2} s(s+1)$, one expects that such matter can only be constructed in terms of Pauli-Lubanski limits

\footnotetext{
${ }^{2}$ String-local fields are not objects of String Theory. The latter is a quantization theory of world surfaces whose quantum content bears no relation to causal localization in Minkowski spacetime and Wigner particles.
} 
$m \rightarrow 0, s \rightarrow \infty$ with $\kappa$ fixed, but fortunately it turned out that the use of modular localization theory permits a more direct explicit constructions of sl fields [5].

Our main result consists in providing arguments which support the impossibility of quantum field theoretical interactions between WS with normal matter. This leaves this kind of sl matter with an aura of mystery even more than seven decades after the discovery of its representational description by Wigner; for this reason we will refer to these representations and the associated sl fields briefly as the "Wigner stuff" (WS).

For a long time the WS representation class did not reveal its quantum field theoretic localization properties. The standard group theoretical covariantization method to construct intertwiners [2] which convert Wigner's unitary representations into their associated $\mathrm{pl}$ quantum fields does not work for the WS representations. Hence it is not surprising that attempts in [6] (and more recently in [7]) which aim at the construction of covariant wave functions and associated Lagrangians fell short of solving the issue of localization. In fact an important theorem [8] dating back to the 70s revealed that it is not possible to associate $\mathrm{pl} \mathrm{Wightman} \mathrm{fields} \mathrm{with}$ these representations.

Using the concept of modular localization, Brunetti, Guido and Longo showed that WS representations permit to construct subspaces which are "modular localized" in arbitrary narrow space-like cones [9] whose core is a semi-infinite string. Modular localization of relativistic wave functions anticipates the causal localization of the related free fields, but it does this in a way which is compatible with the positive energy restriction. As a result it is very different from the quantum mechanical Born localization which describes the dissipation of wave packets and plays an important role in the large-time behavior of quantum fields which is the basis of scattering theory and relates interacting fields with Wigner particles.

In subsequent work $[5,10]$ such generating string-local covariant fields were constructed in terms of modular localization concepts. In the same paper attempts were undertaken to show that such string-local fields cannot have pl composites. These considerations were strengthened in [11]. A rigorous proof which excludes the possibility of finding compact localized subalgebras (generated by test-function-smeared pl fields) was finally presented in a seminal paper by Longo et al. [4].

Being a positive energy representation one would naively expect that WS shares its stability property and also its ability to couple to gravity through its stress-energy tensor with the other two positive energy classes. Whereas it is certainly not possible to calculate induced gravitational backreaction in terms of semiclassical approximations of the EinsteinHilbert equation without a good understanding of higher spin conserved and trace-free stress-energy tensors, it is also true that the problem of backreaction goes significantly beyond that of constructing higher spin stress-energy tensors. Trial calculations of induced backreactions exist for $s=0,1 / 2$ in a symmetric Robertson-Walker background [12].

The existence of gravitational backreaction is a prerequisite for proposing higher spin matter as potential candidates of dark matter. In view of the sl nature of its generating fields one expects that they leave their substantial mark on the putative gravitational backreaction.

The other requirement which candidates for dark matter must fulfill in order to be consistent with astrophysical data as well as with ongoing earthly particle detection experiments is their low reactivity with respect to quantum matter. Here the non-gravitational inertness of WS (Sects. 3 and 4) becomes important. In fact the new sl renormalization setting suggests that this may be a general property of even massive higher spin matter above a critical spin. Additional calculations which could resolve this problem are on the way.

The aim of the present paper is to convert the question of whether nature uses WS matter into a problem of particle theory. Since the task of local quantum physics (LQP) is to explain properties of matter in terms of the causal localization principles [13], one must show that the lack of reactivity of WS is a consequence of its intrinsically noncompact causal localization.

In the context of quantum theory these principles are much more powerful than their Faraday-Maxwell-Einstein classical counterparts. The concept of modular localization permits to address structural problems of QFT in a completely intrinsic way thus avoiding the use of "fieldcoordinatizations". An illustration of the power of this relatively new concept is the proof of existence of a class of two-dimensional models starting from the observations that certain algebraic structures in integrable $d=1+1$ models can be used to construct modular localized wedge algebras [14]. In the work of Lechner and others this led to existence proofs for integrable models with nontrivial short distance behavior together with a wealth of new concepts (see the recent reviews $[15,16]$ and the references therein).

In renormalized perturbation theory modular localization has become useful in preliminary attempts to replace local gauge theory in Krein space by positivity preserving stringlocal fields in Hilbert space [17,18]. In [9] it was essential to extract localization properties directly in the form of modular localized subspaces; Weinberg's method of constructing the intertwiners of local fields via group theoretical covariance requirements does not work for WS.

In an unpublished previous note [19] the problem of a possible connection between WS and dark matter was addressed. But the recent gain of knowledge from modular localization regarding attempts to unite WS with normal matter under the conceptual roof of AQFT in [4], as well as new insights coming from preliminary perturbative studies of couplings 
involving string-local fields $[17,18]$, led to a revision of these ideas.

In [4] it was shown that the attempt to unite normal matter (matter as we know it) together with WS in a nontrivial way $^{3}$ under the conceptual roof of algebraic QFT (AQFT) leads to an unexpected loss of the so-called "Reeh-Schlieder property" for compact localized observable algebra. The R$\mathrm{S}$ property states that the set of state vectors obtained by the application of operators from a compact localized subalgebra of local observables to the vacuum is "total" in the vacuum Hilbert space [13]. The possibility to change large distance properties of states in the vacuum sector by applying operators localized in a compact spacetime region $\mathcal{O}$ to the vacuum is considered to be a universal manifestation of vacuum polarization.

The R-S property of the observable algebra plays an important role in the Doplicher-Haag-Roberts (DHR) superselection theory [13] which leads to the concept of inner symmetries and Bose/Fermi statistics (absence of para-statistics). The WS fields have no associated observable algebra for which they generate superselection sectors. Whereas finite $s \geq 1$ spin fields become sl only as a result of their interaction, the WS matter is sl in the absence of interactions and it is this property which accounts for its lack of reactivity.

There exists a new renormalization theory for $s \geq 1$ finite spin matter which uses the fact that any massive higher spin free field whose short distance dimension increases with spin $d_{\text {sd }}=s+1$ can be written as a linear combination of its sl counterpart with $d_{\text {sd }}=1$ and derivatives of so-called "escort" fields where all of these free fields depend on the same degrees of freedom in terms of the Wigner spin $s$ creation and annihilation operators $a^{\#}\left(p, s_{3}\right)$. Sl renormalizability requires that the nonrenormalizable pl interaction density $L^{P}$ may be written as a sum $L^{P}=L+\partial^{\mu} V_{\mu}$ where the sl interaction density remains within the power-counting bound (PCB) $d_{\text {sd }}(L) \leq 4$ and the singular $d_{\text {sd }}>4$ contributions are collected in the $\partial V$ contribution which can be disposed of in the adiabatic limit.

This idea, revolutionary (renormalizability-preserving) but at the same time conservative (no violation of causal localization and positivity), is the perturbative realization of a result which has been known for a long time namely that in theories with local observables and a mass gap there always exist interpolating operators of particles which are localized in arbitrary narrow cones whose cores are semiinfinite space-like strings [20].

In the standard formulation of QFT, where localized operators are obtained by smearing fields with test functions, the generating fields of such operators are sl Wightman fields

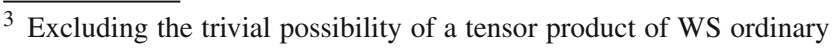
matter.
}

$\Psi(x, e)$ to be smeared in $x$ as well as in the $d=1+2$ de Sitter space of space-like directions $e$ ). The present paper presents some low order illustrations of this perturbation theory and shows that without interacting sl fields there can be no positivity-maintaining renormalization theory involving $s \geq 1$ fields.

The present paper presents arguments which strongly suggest that these conditions cannot be fulfilled in couplings of WS to normal matter. Part of the problem is that the scalar WS fields which were constructed by using modular localization theory have not been obtained in the Pauli-Lubanski limit in which the mass approaches zero while the spin increases so that the Pauli-Lubanski invariant $\kappa^{2}=m^{2} s(s+$ 1) remains fixed.

This leaves the conclusion that, apart from gravitational backreaction which require the solution of additional renormalization problems of the stress-energy tensor ${ }^{4}$ (the substitute for the vacuum-based Wick-product [12]), WS cannot interact with normal matter in a way which complies with the causal localization structure of QFT. In mathematical terminology, WS matter tensor-factorizes with normal matter and the standard properties (including the ReehSchlieder cyclic action of local subalgebras on the vacuum vector [13]) hold only in the tensor factor of normal matter.

The next section presents a "crash course" on Wigner's theory of positive energy representations of the Poincaré group including the explicit construction of sl WS free fields and their two-point functions. In contrast to recent attempts to access higher spin QFTs by Lagrangian quantization [21] and in this way open Pandora's box of indefinite metric and ghosts, Wigner's classification of all positive energy representation is free from quantization "crutches" (quantum positivity has no classical counterpart). These properties are preserved in Weinberg's construction of intertwiners between unitary and the field theoretic $m>0$ representations [2] and the (at that time still) missing massless potentials have been completed by the construction of covariant sl intertwiners in which the understanding of the localization of WS played an important role [5].

The third section highlights an important restriction on renormalizable couplings involving sl fields which is necessary to avoid higher order total delocalization.

Section 4 presents new concepts which are expected to be useful in future model studies of higher spin $s \geq 2$ interactions. The problems to fulfill these requirement grow with increasing spin and suggest the existence of a critical spin above which matter becomes "inert".

\footnotetext{
${ }^{4}$ Whenever the purpose for the construction of $T_{\mu \nu}$ is not to obtain the generators of the Poincaré symmtery but rather the currents for the right hand side of the Einstein-Hilbert equations its is more appropriate to use the terminology "stress-energy tensor".
} 
Section 5 addresses the problem of the role of sl localization in the construction of the correct energy-momentum tensor for higher spin quantum fields which is the prerequisite for the Einstein-Hilbert gravitational coupling.

The concluding remarks point at problems arising from the identification of WS with dark matter.

\section{Matter as we (think we) know it and Wigner's infinite spin "stuff"}

The possible physical manifestations of WS matter can only be understood in comparison to normal matter. Hence before addressing peculiarities it is necessary to recall the localization properties of free massive and finite helicity zero mass fields.

It is well known that all $\mathrm{pl}$ massive free fields can be described in terms of matrix-valued functions $u(p)$ which intertwine between the creation/annihilation operators of Wigner particles and fields [2]. Their associated covariant fields are of the form

$$
\begin{aligned}
\psi^{A, \dot{B}}(x)= & \frac{1}{(2 \pi)^{3 / 2}} \int\left(\mathrm{e}^{i p x} u^{A, \dot{B}}(p) \cdot a^{*}(p)\right. \\
& \left.+\mathrm{e}^{-i p x} v^{A, \dot{B}}(p) \cdot b(p)\right) \frac{\mathrm{d}^{3} p}{2 p_{0}}
\end{aligned}
$$

The intertwiners $u(p)$ and their charge-conjugate counterpart $v(p)$ are rectangular $(2 A+1)(2 B+1) \otimes(2 s+1)$ matrices which intertwine between the unitary $(2 s+1)$-component Wigner representation and the covariant $(2 A+1)(2 B+1)$ dimensional spinorial representation (suppressed indices) labeled by the semi-integer $A, \dot{B}$ which characterize the finite dimensional representations of the covering of the Lorentz group $S L(2, C)$ (the "undotted and dotted" representations). The $a^{\#}(p), b^{\#}(p)$ refer to the Wigner particle and antiparticle creation/annihilation operators and the dot - denotes the scalar product in the $2 s+1$ dimensional spin space.

For a given physical spin $s$ there are infinitely many spinorial representation indices of the homogeneous Lorentz group; their range is restricted by [2]

$|A-\dot{B}| \leq s \leq A+\dot{B}, \quad m>0$

For explanatory simplicity we restrict our subsequent presentation to integer spin $s$; for half-integer spin there are similar results.

All fields associated with integer spin $s$ representation can be written in terms of derivatives acting on symmetric tensor potentials $(A=B)$ of degree $s$ with lowest short distance dimension $d_{\text {sd }}^{s}=s+1$. For $s=1$ one obtains the divergenceless $\left(\partial \cdot A^{P}=0\right)$ Proca vector potential $A_{\mu}^{P}$ with $d_{\mathrm{sd}}=2$, whereas for $s=2$ the result is a divergence- and traceless symmetric tensor $g_{\mu \nu}$ with $d_{\mathrm{sd}}=3$. The terminology "Proca" is used for all tensors.
Free fields can also be characterized in terms of their twopoint functions whose Fourier transformation are tensors in momenta instead of intertwiners. For $s=1$ one obtains (P stands interchangeably for "Proca" or "point-like")

$\left\langle A_{\mu}^{P}(x) A_{\nu}^{P}\left(x^{\prime}\right)\right\rangle=\frac{1}{(2 \pi)^{3}} \int \mathrm{e}^{-i p\left(x-x^{\prime}\right)} M_{\mu \nu}^{P}(p) \frac{\mathrm{d}^{3} p}{2 p^{0}}$,

$M_{\mu \nu}^{P}(p)=-g_{\mu \nu}+\frac{p_{\mu} p_{\nu}}{m^{2}}$

$A_{\mu}^{P}(x)=\frac{1}{(2 \pi)^{3 / 2}} \int \mathrm{e}^{i p x} u_{\mu}(p) \cdot a^{*}(p) \frac{\mathrm{d}^{3} p}{2 p^{0}}$,

$u_{\mu}\left(p, s_{3}\right)=e_{\mu}^{\left(s_{3}\right)}$ polarization vectors

and for higher spin the $M^{\prime} s$ are formed from products of $M_{\mu \nu}^{P}(p)$ according to the combinatorial rules for constructing symmetric and trace-free spin $s$ tensors $A_{\mu_{1} . . \mu_{s}}^{P}$ whose little Hilbert space is a $2 s+1$ dimensional subspace $\mathcal{C}^{2 s+1}$ of the $s$-fold tensor product.

For $m=0$ and finite integer helicity $h$ the two dimensional $\pm|h|$ helicity representation replaces the $2 s+1$ component spin. Despite this difference, the covariant fields turn out to be of the same form as Eq. (1), except that (2) is now replaced by the more restrictive relation

$|A-\dot{B}|=|h|, \quad m=0$,

which excludes tensor potentials but preserves their field strengths with of tensor degree $2|h|$ and $d_{\text {sd }}=|h|+1$ with mixed symmetry properties. This is well known in the case of $|h|=1$ where there exists no massless pl vector potential with $A=1 / 2=\dot{B}$ whose curl is associated to the electromagnetic field strength with $|A-B|=1$.

The cause for the absence massless tensor potentials in (5) is a clash between pl localization and Hilbert space positivity. Gauge theory formally resolves this by allowing compensations between positive contributions from intermediate states with $d_{\mathrm{sd}}$-reducing negative parts. This use of negative probability contributions amounts to the replacement of the positive metric Hilbert space by an indefinite metric Krein space.

Strictly speaking gauge "symmetry" is not a bona fide symmetry but rather a device to extract a physical subtheory of local observables and obtain a perturbative prescription for a unitary $S$-matrix at the price of losing the interpolating fields which relate the causal localization principles with particles. Since positivity has no natural place in classical field theory its presence in the Lagrangian quantization formalism needs special care in particular if higher spins $s \geq 1$ are involved.

The use of Wigner's unitary representation theory for the construction of covariant fields in terms of intertwiner functions avoids these problems. It fails for good reasons in the 
case of massless helicity $|h| \geq 1$ potentials but permit the construction of their associated field strengths.

In fact, it is precisely this absence of massless pl potentials which reveals a basic interrelation between causal localization and positivity whose foundational understanding provides the means to overcome the problem of missing massless potentials. This zero mass helicity problem is interconnected with the violating of the power-counting bound $d_{\mathrm{sd}}(L) \leq 4$ of renormalizability which one runs into if the Lorentz invariant interaction density $L$ contains spin $s \geq 1$ potentials with the (positivity preserving) short distance scaling dimension. ${ }^{5}$ $d_{\mathrm{sd}}=s+1$.

What connects the short distance scaling degree $d_{\mathrm{sd}}=$ $s+1$ of tensors with the singular behavior $m^{-s}$ of their massless limit ( $m^{-s+1 / 2}$ for spinor-tensors) is that the classical dimension in mass units (the "engineering" dimension) is $d_{\text {eng }}=1$ for tensors and $d_{\mathrm{eng}}=3 / 2$ for spinor tensors. Both phenomena, the absence of massless pl potentials and the breakdown of renormalizability in the presence of massive $s \geq 1$ potentials, are interrelated in the sense that a clash between causal localizability and positivity causes for.

A breakdown of renormalizability arises whenever the interaction density violates the power-counting bound $d_{\text {sd }}(L)$ $\leq 4$. Clearly the presence of a tensor potential with $d_{\mathrm{sd}} \geq 2$ always causes such a violation. Abandoning positivity leads to gauge theory which significantly reduces the physical range but still preserves the perturbative construction of a unitary $S$-matrix at the expense of having to cope with additional unphysical degrees of freedom.

Ignoring unphysical aspects of gauge theory one achieves at least a formal solution of the two interconnected problems. But physically it remains a disaster since important physical objects as an electric current of a charge-carrying $s \geq 1$ potential as well as an $s \geq 2$ energy-momentum tensor are not available in gauge theory. This is the basis of the Weinberg-Witten no-go theorem [22]). It is also enters the degree of freedom counting in passing from the massive spin degree of freedom to their massless helicity limits (the van Dam-Veltman-Zakharov discontinuity $[23,24])$. The shortcomings in renormalized perturbation theory attracted less attention since a perturbative prescription for a unitary $S$-matrix preserved at least some of the predictive power in the application of gauge theory to the Standard Model.

The interrelation between positivity and localization suggests to look for a remedy by keeping positivity which secures the important quantum theoretical probability interpretation and rather weaken the pl causal localization. The sl localiza-

\footnotetext{
5 The scaling dimension $d_{\mathrm{sd}}$ characterizes the leading short distance singularity $\lambda^{-2 d_{\mathrm{sd}}}$ of the two-point function under short distance scaling $x-y \equiv \xi \rightarrow \lambda \xi$.
}

tion of WS suggests that the positivity-maintaining Wigner representation theory may achieve this in the form of covariant localization on space-like half-lines.

As Weinberg [2] obtained pl intertwiners by group theoretical arguments based on covariance, one may construct sl intertwiners using the powerful concept of modular localization [5]. For finite spin sl fields there is a simpler way by integrating $\mathrm{pl}$ fields over space-like half-lines with direction $e, e^{2}=-1$. In this way one obtains instead of (3) the slightly more involved formula

$$
\begin{aligned}
M_{\mu \nu}^{s}\left(p ; e . e^{\prime}\right)= & -g_{\mu \nu}-\frac{p_{\mu} p_{\nu} e \cdot e^{\prime}}{(p \cdot e-i \varepsilon)\left(p \cdot e^{\prime}+i \varepsilon\right)} \\
& +\frac{p_{\mu} e_{\nu}}{p \cdot e-i \varepsilon}+\frac{p_{\nu} e_{\mu}^{\prime}}{p \cdot e^{\prime}+i \varepsilon}
\end{aligned}
$$

Clearly the degree of divergence has been lowered from $d_{\mathrm{sd}}^{P}=$ 2 of the Proca potential to $d_{\mathrm{sd}}=1$ for its sl counterpart whereas the vanishing of the divergence has been lost apart from the zero mass limit when $p^{2}=0$.

A newly gained property of (6) is the transversality in $e$ and $e^{\prime}$, which looks like the axial gauge condition but plays a very different role in the present context; instead of defining a global gauge-fixing condition it is the result of a fluctuating spacetime variable $e$, which together with the point of origin $x$ of the string participates in the Lorentz transformations. Those unmanageable ultraviolet and infrared properties which led to the abandonment of $e$ as a gaugefixing parameter turn out to be a blessing in the new interpretation of $e$ as a fluctuating space-like direction of an sl field.

The preservation of positivity is seen by verifying that this formula is obtained from the positivity-obeying Proca field by first defining the associated field strength and then integrating along a half-line in space-like direction $e, e^{2}=$ $-1$

$$
\begin{aligned}
& A_{\mu}(x, e):=\int_{0}^{\infty} \mathrm{e}^{v} F_{\mu \nu}(x+\lambda e) \mathrm{d} s, \\
& F_{\mu \nu}:=\partial_{\mu} A_{\nu}^{P}-\partial_{\nu} A_{\mu}^{P}
\end{aligned}
$$

The $p e \pm i \varepsilon$ factors in (6) result from integrating the Heavyside step function multiplied with $\exp i \lambda p e^{\prime}$, respectively, with exp $-i \lambda p e$. This integration causes sl fields to be distributions as boundary values of analytic functions in $e$ [5].

The Proca potential and its sl counterpart share the same field strength, so their difference according to the Poincaré lemma must be a gradient of an sl scalar field which turns out to be

$$
\begin{aligned}
\phi(x, e) & :=\int_{0}^{\infty} \mathrm{d} \lambda \mathrm{e}^{\mu} A_{\mu}^{P}(x+\lambda e) \\
& =\frac{1}{(2 \pi)^{3 / 2}} \int\left(\mathrm{e}^{i p x} u(p, e) \cdot a(p)+\text { h.c. }\right) \frac{\mathrm{d}^{3} p}{2 p_{0}}
\end{aligned}
$$




$$
\begin{aligned}
u(p, e) & :=u(p)_{\mu} \mathrm{e}^{\mu} \frac{1}{i(p \cdot e+i \varepsilon)}, \\
M^{\phi, \phi} & =\frac{1}{m^{2}}-\frac{e \cdot e^{\prime}}{(p \cdot e-i \varepsilon)\left(p \cdot e^{\prime}+i \varepsilon\right)}
\end{aligned}
$$

with the conjugate intertwiner being the complex conjugate of $u$. The intertwiner of the sl vector potential can be red off from the linear relation between the two vector potentials (suppressing again the $s_{3}$ indices)

$$
\begin{aligned}
& A_{\mu}(x, e)=A_{\mu}^{P}(x)+\partial_{\mu} \phi, \\
& u_{\mu}(p, e)=u_{\mu}(p)-i p_{\mu} u(p, e) .
\end{aligned}
$$

The scalar sl fields $\phi$ will be referred to as "escorts". This terminology intends to highlight the fact that, unlike the negative metric Stückelberg fields and the ghost fields of gauge theory, they do not enlarge the degrees of freedom. In a somewhat metaphoric terminology they may be described as objects which result from applying Occam's razor to gauge theory. One new aspect of the sl formalism is the appearance of off-diagonal contributions between the potentials and its escorts.

The localization of these new sl fields on space-like strings ${ }^{6}$ (or rays) $\mathcal{S}(x, e)=x+\mathbb{R}_{+} e$ can be seen from (7); contrary to infinite spin WS one does not need to appeal to modular localization theory. In fact (although not clearly stated in [5]) by allowing different measure $\mathrm{d} \mu(\lambda)$ one can obtain the continuous set of all finite spin fields with any prescribed short distance dimensions $d_{\mathrm{sd}} \geq 0$. Together with their pl siblings they form the linear part of the local equivalence (Borchers) class of relatively local fields.

Interestingly the equivalence class for sl massless helicity fields is a smaller discrete set for which (5) is replaced by $h \leq|A+B|$ [25]. This indicates that only those massive sl fields which have a smooth massless limit are useful. There exists also an inverse process of passing from massless helicity fields to their massive spin $s=|h|$ counterpart previously called the "fattening" of massless helicity fields to their massive counterpart. This enhancement of the degree of freedom from the two helicities to the $2 s+1$ degrees of freedom with $s=|h|$ is owing to the fact that the positivitymaintaining mass deformation of the dispersion $p_{0}=\omega_{m}(p)$ diminishes the number of null-states (viewing the momentum space two-point function $M$ as an inner product) and in this way increases the number of independent states.

For the case at hand the "fattening of the photon" just means passing from $p \in \partial V_{+}$to $p \in V_{+}$in (6). From the massive two-point function one can construct the massive free vector potential according to the Jost-Schroer theorem [26].

\footnotetext{
${ }^{6}$ Another option which seems to lead to some simplification of the formalism consists in using light-like strings.
}

The massless scalar field $\varphi$ obtained as $\lim _{m \rightarrow 0} m \phi(x, e)$ $=\varphi(x)$ is the massless relic of the $s_{3}=0$ spin component. Ascribing the fattening of the photon to a spontaneous symmetry breaking is a misunderstanding. The role which the new SLF setting ascribes to the Higgs particle is much deeper (next section).

That a comparatively mild relaxation of causality from pl to sl as required by positivity can generate unexpected changes within the conceptual setting of QFT is somewhat surprising. There exist formulations which do not rely on the classical Lagrangian formalism as e.g. the Epstein-Glaser setting of "causal" renormalized perturbation theory [3]. The SLF formulation which places the preservation of positivity and the related weakening of causal localization into the forefront is the most recent step in letting QFT stand on its own feet.

Lagrangian quantization does not reveal these interconnections and hence is not a reliable guide for solving actual problems. The classical formalism suggests the form of conserved currents or energy-momentum tensors and permits the verification of the correct commutation relations for low spin within the canonical formalism. But in the presence of higher spins one needs the explicit form of the higher spin quantum fields and their two-point functions and this requires their positivity-maintaining Wigner-Weinberg construction [2]; the use of higher spin Lagrangian formalism with its additional auxiliary fields and their unclear physical interpretation is not a trustworthy substitute.

The $s \geq 1$ zero mass and renormalization problem can be resolved in two ways. One way imposed by the Lagrangian quantization is to sacrifice positivity and be content with a restricted physical range offered by gauge theory; the only advantage is that one is able to do this within the standard formalism of the books on QFT. It needs the presence of additional phantom (ghost) degrees of freedom but this can be incorporated into the Feynman rules or the euclidean functional integral representation so that the rules just look like an extension of those for $s</ 1$ interactions.

The discovery of the sl localization of the fields associated to WS suggested a better way, which lets positivity determine the tightest covariant causal localization with which it can be consistent. This turns out to be localization on semi-infinite space-like strings $x+\mathbb{R}_{+} e, e^{2}=-1$. The new kinematical aspects were presented for $s=1$. The main difference is that one obtains a linear relation between the $d_{\mathrm{sd}}=2 \mathrm{pl}$ Proca vector potential and its better behaved $d_{\mathrm{sd}}=1 \mathrm{sl}$ sibling $A_{\mu}(x, e)$ in which a sl escort field $\phi$ enters.

The formal similarity of the escort $\phi$ with the negative metric Stückelberg field [27] should not allow one to overlook the enormous conceptual difference. Sl escorts are degrees of freedom-maintaining natural objects which linearly connect the pl Proca field with its sl counterpart. Their $d_{\text {sd }}=2$ 
derivative $\partial \phi$ compensates for the leading contribution of the $d_{\mathrm{sd}}=2$ Proca potential and converts it into the better behaved $d_{\mathrm{sd}}=1 \mathrm{sl}$ potential. It is important to note that the sl potentials with $d_{\mathrm{sd}}=1$ are precisely those which (according to a previous remark) admit massless helicity limits.

These conversions of integer spin fields from $\mathrm{pl}$ to $\mathrm{sl}$ (and with appropriate modifications also for $s>1$ Fermi-fields) can be extended to $s \geq 1$ Proca tensor potentials of arbitrary rank [28-30]. Instead of $A_{\mu}^{P}$ one starts from a divergence- and trace-free symmetric tensor potentials $A_{\mu_{1} \ldots \mu_{s}}$ of tensor rank $s$. Iterated integration along a space-like direction $e$ leads to $s$ symmetric traceless and divergence-less sl $\phi$ tensor escorts of lower rank,

$$
\begin{aligned}
\phi_{\mu_{1} \ldots \mu_{k}}(x, e)= & \int \mathrm{d} \lambda_{1} \ldots \mathrm{d} \lambda_{s-k} e^{\nu_{1}} \ldots e^{\nu_{s-k}} A_{\nu_{1} \ldots \nu_{s-k}, \mu_{1} \ldots \mu_{k}}^{P} \\
& \times\left(x+\lambda_{1} e+\ldots+\lambda_{s-k} e\right) .
\end{aligned}
$$

Again one can describe these escorts in terms of their $e$ dependent intertwiner functions. The linear relation between the sl potential containing the symmetrized derivatives of the tensorial escorts now reads

$$
\begin{aligned}
A_{\mu_{1}}, \ldots, \mu_{s}(x, e)= & A_{\mu_{1}}^{P}, \ldots, \mu_{s}(x) \\
& +\operatorname{sym} \sum_{k=1}^{s} \partial_{\mu_{1}} \ldots \partial_{\mu_{k}} \phi_{\mu_{k}+1 \ldots \mu_{s}} .
\end{aligned}
$$

The definition of the sl tensor potentials in terms of the degree $2 s$ field strength tensors is the analog of (7) with the field strength defined in terms of the Proca tensor potential as

$$
\mathcal{F}_{\mu_{1}, \ldots \mu_{s}, \nu_{1} \ldots \nu_{s}}=\text { as }(\mu \leftrightarrow \nu) \partial_{\mu_{1} \ldots \partial_{\mu_{s}}} A_{\nu_{1} \ldots \nu_{s}}^{P}
$$

As a result of the symmetry of $A_{\nu_{1}, . . \nu_{s}}^{P}$ the antisymmetrization needs only to be done pairwise in $\mu_{i}-v_{i}$ for every $i$. By an extension of the Poincaré lemma to higher differential forms the difference of the sl potential and its pl counterpart is again of the form of derivatives on lower tensor escorts which in turn have the form of iterated line integrals [30].

The sl field strengths defined in this way in terms of the Proca potentials for $m \rightarrow 0$ are not those which one obtains from the Weinberg intertwiner construction starting from Wigner's massless $|h|=s$ representation. They contain admixtures of all $|h| \leq s$. The escort formalism is flexible enough to steer the amount of smaller helicity admixtures by using the fact that there are different massive sl potentials which describe the $2 s+1$ massive degrees; in particular it permits one to identify a specific massive sl tensor potential whose massless limit contains only the highest helicity contribution. This flexibility of string-localization is important for the avoidance of the before mentioned van Dam-Veltman-Zakharov discontinuity and also allows a profound understanding for circumventing the WeinbergWitten no-go theorem. Both problems are artificially created by the use of the restrictive $\mathrm{pl}$ intertwiners in passing from Wigner's representation theory to covariantly localized fields.

Even more startling is the "fattening formula" which expresses the two-point function of the massive Proca potential with its $2 s+1$ degrees of freedom in terms of that of the massless helicity $|h|=s$ sl potential (or even its massless field strengths of tensor degree $2 s$ ). This flexibility between inequivalent positive energy Wigner representations is not directly visible in Wigner's group theoretic formalism it rather requires the description of its intrinsic modular localization [9] in the form of sl fields.

Before commenting on perturbative renormalized perturbation theory it is helpful to collect those properties which make the sl higher spin fields useful.

- Whereas pl massive tensor potentials have short distance dimension $d_{\mathrm{sd}}=s+1$, their sl counterparts have $d_{\mathrm{sd}}=1$ independent of $\operatorname{spin}\left(d_{\mathrm{sd}}=3 / 2\right.$ for half-integer spin). Hence there are always first order sl interaction densities of maximal polynomial degree 4 within the power-counting limit $d_{\mathrm{sd}}^{\text {int }} \leq 4$, Under certain conditions their use in renormalization theory converts nonrenormalizable $\mathrm{pl}$ interactions into sl renormalizable ones (Sect. 4). This shows that renormalizability is closely related to a weakening of causal localizability which in turn is required by upholding positivity.

- S1 tensor potentials and their composites as conserved currents and energy-momentum tensors have smooth $m \rightarrow 0$ limits. The Weinberg-Witten no-go theorem for $s \geq 1$ massless conserved currents and $s \geq 2$ conserved energy-momentum tensors [22] can be overcome (Sect. 5) by converting the corresponding massive expressions into their sl counterparts before taking their massless limits. The smooth passing from massive sl spin fields to their massless helicity counterparts also solves the so-called van Dam-Veltman-Zakharov discontinuity problem $[23,24]$ which one encounters in the $\mathrm{pl}$ description of massive versus massless gravity $[28,29]$.

- The preservation of positivity in $s=1$ couplings leads to an interesting contrast with GT whose physical range is restricted to gauge invariant local observables and the perturbative calculation of the globally gauge invariant $S$-matrix. The physical interpolating fields whose largetime asymptotic behavior relate the causal localization principles of QFT with the analytic properties of scattering amplitudes of particles is outside the physical range of GT. The description of the reduced physical content of GT requires the use of a rather large number of unphys- 
ical indefinite metric and ghost degrees of freedom in the perturbative calculations which, similar to catalyzers in chemical processes, disappear in the calculated gauge invariant quantities. Whereas the improvement of short distance- and zero mass-properties for $s \geq 1$ free pl potentials in GT are enforced by compensating parts of positive probabilities of quantum theory by negative contributions from negative metric fields and ghosts, the SLF achieves the reduction of $d_{\mathrm{sd}}=s+1$ to $d_{\mathrm{sd}}=1$ by the use of the less tight sl causal localization.

The prerequisite for the use of gauge theory is the existence of local observables, however, as mentioned already in the introduction, the sl fields which generate the infinite spin WS matter have no compactly localized observables. It is not possible to construct such fields in terms of Weinberg's group theoretic method of constructing intertwiners based on the conversion of Wigner's unitary representations into covariant representations of the Lorentz group (which automatically leads to $\mathrm{pl}$ fields). Rather one is required to use the more recent intrinsic characterization of causal localization in terms of modular localization [5]. Whereas intertwiner functions $u(p, e)$ for finite spin fields and their two-point functions are rational in $p(6)(8)$, the corresponding functions WS fields for $s \rightarrow \infty$ at a fixed value of the Pauli-Lubanski invariant $\kappa^{2}=m^{2} s(s+1)$ are transcendental.

A particular WS intertwiner with simple small and large momentum space behavior has been given in terms of an exponential function in [11]

$u(p, e)(k)=\exp i \frac{\mathbf{k}\left(\mathbf{e}-\frac{p_{-}}{e_{-}} \mathbf{p}\right)-\kappa}{p \cdot e}$

here $k$ is a two-component vector of length $\kappa$; the Hilbert space on which Wigner's little group $E(2)$ acts consists of square integrable functions $L^{2}\left(k, \mathrm{~d} \mu(k)=\delta\left(k^{2}-\kappa^{2}\right) \mathrm{d} k\right)$ on a circle of radius $\kappa$. The vector arrow on $e$ and $p$ refer to the projection into the 1-2 plane, and the $e_{-}, p_{-}$refer to the difference between the third and zeroth component. The most general solution of the intertwiner relation differs from this special one by a function $F(p \cdot e)$ which is the boundary value of a function which is analytic in the upper half-plane [5].

The two-point function is clearly a $J_{0}$ Bessel function. The calculation in [11] was done in a special system. Writing its argument in a covariant form one obtains ${ }^{7}$

\footnotetext{
${ }^{7}$ I am indebted to Henning Rehren for showing me the covariantization of Köhler's result.
}

$$
\begin{aligned}
M^{W S}(p, e) \sim & J_{0}(\kappa|w(p, e)|) \\
& \times \exp -i \kappa\left(\frac{1}{p \cdot e-i \varepsilon}-\frac{1}{p \cdot e^{\prime}+i \varepsilon}\right) \\
\text { with } w^{2}(p, e) & =-\left(\frac{e}{e \cdot p-i \varepsilon}-\frac{e^{\prime}}{e^{\prime} \cdot p+i \varepsilon}\right)^{2} .
\end{aligned}
$$

The exponential factor compensates the singularity of $J_{0}$ at $e \cdot p=0$. Note that the Pauli-Lubanski invariant $\kappa$ has the dimension of a mass so that the argument of the two-point function of the sl field has the correct engineering dimension $d_{e n}=1$ of a bosonic quantum field.

The main purpose of this somewhat academic calculation is to convince the reader that there are explicitly known transcendental WS intertwiner and two-point functions whose associated propagators have a well-behaved ultraviolet and infrared behavior. As already mentioned, the physical reason why these fields are nevertheless excluded from appearing in interaction densities is that there are good reasons to believe that higher order perturbation theory lead to a complete delocalization; this issue will be explained in the next section.

\section{The problem of maintaining higher order sl localization}

It is well known that the condition for renormalizability in the case of $s<1$ interactions is the power-counting bound of the interaction density $d_{\mathrm{sd}}^{\text {int }} \leq 4$. Since the minimal short distance dimension of positivity preserving $\mathrm{pl}$ spin $s$ fields is $s+1$, there are no positivity preserving pl renormalizable interactions involving $s \geq 1$ fields. Sl free fields on the other hand which possess a massless limit have an $s$-independent short distance behavior $d_{\mathrm{sd}}=1$, so that one can always find polynomials of maximal degree 4 within the power-counting limitation which represent sl interaction densities with a prescribed field content.

However, the use of sl interaction densities does not come without a price. The weakening of causal localization from pl to sl must not affect particles and their scattering amplitudes. Relativistic particles remain what they always were since Wigner classified their wave function spaces; the dissipation of their wave packets is determined by Born's quantum mechanical localization (equivalent to the Newton-Wigner localization), which bears no direct relation to the operational causal localization of quantum fields.

The string-independence of the $S$-matrix, as mentioned before, is guaranteed by a structural theorem of local quantum physics [20] which states that in a theory with local observables and a mass gap there always exist operators localized in arbitrary tight space-like cones (whose cores are semi-infinite space-like strings) which interpolate the particles. In a field theoretic setting the generating fields of such operators must 
be sl; fields with a weaker than sl localization (e.g. localization on space-like hyper-surfaces) are not needed in order to describe the particle content and their scattering amplitudes.

In a perturbative construction of a model which starts with a Lorentz scalar interaction density in terms of products of free fields the $e$-independence of the $S$-matrix must be imposed as an additional condition on the sl interaction density $L$ (which has no counterpart for $s<1 \mathrm{pl}$ interaction) namely the $L$ must be part of a $L, V_{\mu}$ pair with [18]

$d_{e}\left(L-\partial^{\mu} V_{\mu}\right)=0, \quad d_{e}=d e_{\mu} \frac{\partial}{\partial e_{\mu}}$,

using the differential calculus of the $d=1+2$ de Sitter space of space-like directions. The heuristic content of this relation is the preservation of the physical content of the nonrenormalizable $\mathrm{pl}$ interaction density $L^{P}=L-\partial^{\mu} V_{\mu}$ while collecting the most singular contributions above the power-counting bound in the form of a divergence which can then be disposed in the adiabatic limit of the first order $S$-matrix

$S^{(1)}=\int L \mathrm{~d}^{4} x=\int\left(L-\partial^{\mu} V_{\mu}\right) \mathrm{d}^{4} x$.

Since $d_{\text {sd }}^{L} \leq 4$, whereas the pl interaction involves $s \geq 1$ fields which violate the power-counting bound since $d_{\mathrm{sd}}^{L^{P}}>$ 4 , the role of the divergence term is to "dispose the access above 4 at infinity". This "magic" would not work without the intervention of the escort fields. A simple illustration is provided by the sl interaction density $L$ of massive scalar QED

$L=A_{\mu}(x, e) j^{\mu}(x)$.

It is related to its $d_{\mathrm{sd}}^{L^{P}}=5$ power-counting bound violating $\mathrm{pl} \mathrm{counterpart} L^{P}$ as

$L^{P}=A^{P} \cdot j=L-\partial^{\mu} V_{\mu}, \quad V_{\mu}(x, e):=\partial_{\mu} \phi j^{\mu}$,

$A_{\mu}^{P}(x)=A_{\mu}(x, e)+\partial_{\mu} \phi(x, e)$

where the linear relation between $\mathrm{pl}$ and sl potential in terms of the escort field in the second line has been used.

In this way one solves two problems in one stroke, on the one hand one expresses the (first order) $S$-matrix in terms of a renormalizable $d_{\mathrm{sd}}=4$ interaction density, and at the same time the $e$-dependence disappears in agreement with the theorem about interpolating fields whose large-time behavior describes ordinary particles and their scattering amplitudes.

For the $e$-independence of the higher order contributions to the $S$-matrix it is easier to work with a slightly weaker form of the $L, V_{\mu}$ pair condition by viewing the $\phi(x, e)$ as a differential zero form on the $d=1+2$ dimensional directional de Sitter space and defining the one form $u=d_{e} \phi$, which turns out to allow for a massless limit.
In this way the $L, V_{\mu}$ pair condition can be replaced by a $L, Q_{\mu}$ condition. One finds

$d_{e} A_{\mu}=\partial_{\mu} u, \quad u=d_{e} \phi$,

$d_{e}\left(L-\partial^{\mu} V_{\mu}\right)=d_{e} L-\partial^{\mu} Q_{\mu}=0, \quad Q_{\mu}:=d_{e} V_{\mu}$.

$A, \phi, L, V$ are $d_{\mathrm{sd}}=1$ zero-forms whereas $u$ and $Q_{\mu}$ are exact $d_{\mathrm{sd}}=1$ one-forms; together with the exact two-form $\hat{u}$ (8) they exhaust the with $A_{\mu}^{P}$ linear related and relatively local among themselves $d_{\mathrm{sd}}=1$ forms. The $L, Q_{\mu}$ formalism is somewhat weaker than its $L, V_{\mu}$ counterpart. Whereas the latter is a decomposition of a nonrenormalizable pl interaction density into a renormalizable sl $L$ and singular but adiabatically disposable part, the former relates the differential one form $d_{e} L$ with the adiabatically disposable divergence of $Q_{\mu}$. This turns out to be sufficient for securing the on-shell $e$-independence. In contrast to $V_{\mu}$ the $Q_{\mu}$ admits a massless limit.

We will refer to Eq. (20), which expresses the $e$ independence in terms of a closed zero form, as the $L, V$, respectively, $L, Q$ relation; both conditions ensure the first order $e$-independence of $S$. In the sequel we will only use the $L, Q$ condition.

As the independence of $S$ from gauge-fixing parameters in gauge theory setting, this $e$-independence in the Hilbert space setting results from cancellations between different $e$ dependent contributions in the same order. But different from unphysical gauge dependent correlation functions, vacuum expectation values of charge-carrying sl fields are positivitymaintaining extended Wightman functions (i.e. endpoint $x$ and directional $e$-smearing).

In order to secure the $e$ independence in higher orders we must extend the $L, Q_{\mu}$ relation (20) to higher order timeordered products. The second order $L, Q_{\mu}$ pair requirement ${ }^{8}$ reads

$\left(d_{e}+d_{e^{\prime}}\right) \mathrm{TLL}^{\prime}-\partial^{\mu} T Q_{\mu} L^{\prime}-\partial^{\mu^{\prime}} \mathrm{TLQ}_{\mu}^{\prime}=0$.

If it where not for the distributional singularities of $T$ products at coalescent points, this would follow from (20). In those cases where the singular structure of $T$ matters this amounts to a normalization condition which must be implemented. Resulting second order ambiguities are already determined in the one particle contraction component ("tree approximation").

For massive spinor QED the relation is automatically fulfilled in terms of the standard free field propagator. The more singular scalar QED contains $d_{\mathrm{sd}}=2$ derivatives $\partial \varphi$ of the scalar charged field which according to the minimal scaling

\footnotetext{
8 The $Q_{\mu}$ formalism is somewhat simpler than its $V_{\mu}$ counterpart. For massive QED and couplings of massive vector mesons to Hermitian matter ("Hermitian QED", the Higgs abelian model) it is easy to see their equivalence.
} 
rules of the Epstein-Glaser renormalization theory lead to a delta counterterm,

$$
\left\langle T \partial_{\mu} \varphi^{*} \partial_{\nu}^{\prime} \varphi^{\prime}\right\rangle=\partial_{\mu} \partial_{\nu}^{\prime}\left\langle T \varphi^{*} \varphi^{\prime}\right\rangle+c g_{\mu \nu} \delta\left(x-x^{\prime}\right) .
$$

The imposition of Eq. (21) fixes the parameter $c$ with the expected result of an induced second order term of the expected form $\delta\left(x-x^{\prime}\right) A_{\mu} A^{\mu} \varphi^{*} \varphi$.

Note that no arguments of classical gauge theory (as the replacement $\partial \rightarrow D=\partial+i g A$ ) has been used; the result is solely a consequence of the causal localization principles and Hilbert space positivity which require the $e$-independence of particle properties from the sl localization of their interpolating fields.

There are some interesting foundational aspects of this otherwise trivial calculation. The independent fluctuation in $e$ and $e^{\prime}$ do not allow one to set $e=e^{\prime}$ in off-shell correlations (6); the different $i \varepsilon$ prescriptions for $e$ and $e^{\prime}$ in the off-shell propagator prevent this. This singular behavior is reminiscent of that for coalescing points. There are indications that the use of light-like $e^{\prime} s$ may be better in this respect.

The second order on-shell $e$-independence corresponds to the second order gauge invariance of the scattering amplitude; but whereas in gauge theory the individual contributions are gauge dependent but finite, the directional fluctuations in $e$ require cancellations between individual contributions before setting $e=e^{\prime}$ and obtain $e$-independence.

The "magic" of $L, V_{\mu}$ pairs with (20) is that on the one hand they permit to use the lower short distance dimension of sl fields (and in this way lead to $L^{\prime} s$ within the powercounting bound of renormalizability) and on the other hand they also guarantee the $e$-independence of the $S$-matrix since the derivative contributions in (21) disappear in the adiabatic $S$-matrix limit

$\left(d_{e}+d_{e^{\prime}}\right) S^{(2)}=0, \quad S^{(2)} \sim \int \mathrm{TLL}^{\prime}$

The off-shell extension in terms of Bogoliubov's $S(g)$ operator functional with a spacetime dependent coupling leads to correlation functions of interacting sl fields. As the $S$ matrix is independent of the $e^{\prime} s$ of the inner propagators (after summing over sufficiently many contributions in a fixed perturbative order), the correlation functions of interacting fields must only depend on the $e^{\prime} s$ of those fields.

A new phenomenon is that the higher order interactions spread the $e$-dependence also to those fields which entered the first order interaction density as $s<1$ pl fields. ${ }^{9}$ In fact the interacting matter fields in the new sl setting of renormalization theory are in a certain sense stronger sl than the vector potential which already entered $L$ as a sl free field and which remains linearly related with its pl field strengths.

\footnotetext{
9 The perturbation theory of interacting string-local fields is still in its beginnings. A mathematically rigorous presentation for massive QED will be the subject of forthcoming work by Jens Mund.
}

In the limit of massless sl vector mesons the correlation functions change their physical properties; the particle setting in a Wigner-Fock Hilbert space is lost and the strings of the charge-carrying fields become "stiff" (the nonperturbative spontaneously breaking of Lorentz invariance in charged sectors [31]). Little is known about the spacetime manifestations of these physical changes (particles $\rightarrow$ infraparticles) apart from perturbative momentum space prescriptions for photon-inclusive cross sections [32].

The $L, V_{\mu}$ (or $L, Q_{\mu}$ ) pair property (19) is a necessary condition for maintaining sl localization; it permits to sail between the "Scilla of nonrenormalizability and the Charybdis of total de-localization". Heuristically speaking it provides a compensatory mechanism between contributions to the same order which prevents the total de-localization resulting from the integration over $x$ in inner strings $x+\mathbb{R}_{+} e$ in individual Feynman diagrams. The main point of the present work is the argument that with WS fields in $L$ it is not possible to fulfill the $L, Q_{\mu}$ condition so that WS matter can only exist in the interaction-free form. We will refer to such matter as inert (see the next section).

There is another important physical aspect of the $L, Q_{\mu}$ pair property in which the escort field $\phi$ plays an essential physical role even though it does not add new degrees of freedom. Heuristically speaking the transition from long range massless sl vector potentials to their short range massive counterpart is not possible without the appearance of the $\phi$ escort, they (and not the Higgs particles) "fatten" the photon and its higher helicity counterparts. In some models they already appear in the first order interaction density (see Eq. 26).

This is somewhat reminiscent of the presence of the bosonic Cooper pairs in the BCS description of superconductivity; without their presence it is not possible to convert long range classical vector potentials into their short ranged counterparts inside the superconductor. As the $\phi$ in massive QED they are not the result of additional degrees of freedom, they rather arise from rearrangements of existing condensed matter degrees of freedom in the low temperature phase.

The QFT analog of the BCS or the Anderson screening mechanism is the screened "Maxwell charge" [17] i.e.

$$
\begin{aligned}
& j_{\mu}:=\partial^{v} F_{\mu \nu}, \quad Q_{\mathrm{scr}}=\int j_{0}(x) \mathrm{d}^{3} x=0, \text { screening } \\
& \partial^{\mu} j_{\mu}=0, \\
& Q_{\mathrm{SSB}}=\int j_{0}(x) \mathrm{d}^{3} x=\infty, \text { long dist.divergence. }
\end{aligned}
$$

The screening property (first line) only depends on the massive field strength and not on the kind of matter to which it couples (which may be complex or Hermitian matter). This includes non-interacting massive vector mesons for which $j_{\mu}=-m^{2} A_{\mu}^{P}$. Spontaneous symmetry breaking on the other 
hand reveals itself in form of a conserved current whose charge diverges instead of being zero (second line). The normal case of an inner symmetry is that of a nontrivial finite charge.

Renormalizable models are generally uniquely specified in terms of their field content. In the above case of massive scalar QED the form of the first order sl coupling is uniquely fixed by the $L, Q_{\mu}$ pair condition (the preservation of sl localization). The second order pair condition is a normalization requirement which induces the $A \cdot A|\varphi|^{2}$ term.

This induction which in classical QED results from the fibre-bundle structure $\partial_{\mu} \rightarrow D_{\mu}=\partial_{\mu}-i g A_{\mu}$ is in (positivity preserving) QFT a structural consequence of the causal localization principle. Gauge theory hides this important fact by preserving the classical fibre-bundle interpretation at the price of indefinite metric (which does not only violate the probability interpretation but also denaturalizes the physical localization of QFT). The role of gauge symmetry and gauge invariance is to recover part of the lost physical properties for a more restricted gauge invariant subtheory. The induction of the quadratic contribution $A \cdot A|\varphi|^{2}$ is a result of the imposed invariance under "gauge symmetry" which (apart from the fact that this is not a physical symmetry) is equivalent to the classical fibre-bundle requirement.

The new string-local QFT (SLFT) setting retains all physical properties by replacing the mute global gauge-fixing parameters by individually fluctuating local space-like string directions. In this way all the unphysical "dead wood" of indefinite metric Stückelberg fields and ghosts will not be allowed to enter in the first place. The idea that quantum fields should follow the pl localization of classical field theory is too restrictive for constructing interacting higher spin quantum fields.

The sl localization preserving $L, V_{\mu}$ induction becomes much richer if the interaction of $A_{\mu}$ with complex matter is replaced by Hermitian matter (the abelian Higgs model). The application of the $L, V$ requirement to the coupling of a massive vector meson to a Hermitian $H$ field proceeds as follows. The $\mathrm{pl}$ interaction with the lowest short distance dimension $d_{\mathrm{sd}}^{P \text {,int }}=5$ is $L^{P}=m A^{P} \cdot A^{P} H$. Converting it into a sl $L, V_{\mu}$ pair, one obtains [easy to check by the use of the free Klein-Gordon equation for $H$ :

$$
\begin{aligned}
L & =m\left\{A \cdot(A H+\phi \overleftrightarrow{\partial} H)-\frac{m_{H}^{2}}{2} \phi^{2} H\right\} \\
V_{\mu} & =m\left\{A_{\mu} \phi H+\frac{1}{2} \phi^{2} \overleftrightarrow{\partial}{ }_{\mu} H\right\}, \\
L & -\partial V=L^{P}=m A^{P} \cdot A^{P} H, \quad Q_{\mu}=d_{e} V_{\mu} \\
& =A_{\mu} u H+u\left(\phi \partial_{\mu} H-\partial_{\mu} \phi H\right), \quad u=d_{e} \phi
\end{aligned}
$$

In this case the on-shell $e$-independence requirement (21) in second and third order tree approximation leads to a much richer collection of induced terms than for scalar massive QED [17].

Different from scalar QED (17) the form of the $L, V_{\mu}$ pair cannot be immediately red off from $L^{P}$. One obtains the above decomposition by making a separate trilinear Ansatz for $L$ and $V_{\mu}$ and verifying that the requirement $d_{e}(L-\partial V)=$ 0 fixes all parameters and results in Eq. (26).

Whereas in the massive scalar QED model this requirement induces only the $A \cdot A \varphi^{*} \varphi$ term, the induction in the case of an interaction with a Hermitian field leads in addition to the expected $A \cdot A H^{2}, A \cdot A \phi^{2}$ terms (which as in scalar QED can be absorbed into a changed time-ordered product) also to second order induced $H^{4}, \phi^{4}, H^{2} \phi^{2}$ terms (from second order $A-A$ contractions in (21) as well as to an induced $H^{3}$ term $[17,18]$. The second and third order tree approximation suffices to fix their coupling strengths in terms of the three physical parameters of the elementary model-defining $A_{\mu}, H$ fields, namely the coupling strength and (ratios of) the two masses $m, m_{H}$.

The result corresponds to the terms induced by gauge invariance of the $S$-matrix in [33]. It is also the same as that of the formal calculation based on the SSB Higgs mechanism, except that in that case one postulates a Mexican hat potential instead of inducing it from gauge invariance or from causal localization in a positivity preserving sl setting. As soon as vector potentials enter one has to follow the more basic rules of either GT or SLFT.

QFT is a foundational quantum theory in which all physical properties of a model are intrinsic. For the physical interpretation of its content prescriptions which led to its construction are not of much help; one is not forced to rely on prescriptions, the model itself (its fields and correlation functions) contains its own interpretation. In many cases it is the field content alone which determines the form of the renormalized interaction density. For the case at hand the $A, H$ field content and the $L, Q_{\mu}$ renormalization requirement fix the first and second order interaction density, including the $H$ self-interaction which together with the escort field $\phi$ has the shape of a field-shifted Mexican hat potential.

The difference from an SSB situation becomes evident when one looks at the conserved current. In a SSB situation the charge diverges $Q_{\mathrm{ssb}}=\infty$. In fact the nonexistence of a symmetry generator, in spite of the presence of a conserved current $\partial^{\mu} j_{\mu}=0$ which expresses the local charge balance, is the definition of a SSB. The shift in field space on a Mexican hat potential is a way to obtain such a situation if the resulting conserved current has a divergent charge.

This is certainly not the case for the Higgs model because the identically conserved current of the field strength of a massive vector meson (the only conserved current of the Higgs model) is the identically conserved Maxwell current of a massive $F_{\mu \nu}$ which always leads to a screened charge $Q_{\mathrm{sc}}=0$ independent of the nature of the matter (complex 
or Hermitian). Nothing could be physically more apart than $Q_{\text {sc }}=0$ and $Q_{\text {ssb }}=\infty$.

One formal reason why in gauge theory the BRST invariance of the on-shell $S$-matrix which leads to the correct induced $H$ self-interactions is easily confused with the offshell Mexican hat prescription of SSB is that in functional Feynman graph representations it is difficult to distinguish between relations which only hold on-shell from off-shell relations. For this reason it was important to use the causal gauge invariant (CGI) operator Epstein-Glaser formulation $[33,34]$.

There is no problem to extend the construction of the $L, Q$ pair to self-interacting massive vector mesons and calculate the second order induced terms. One finds that there is an uncompensated $d_{\mathrm{sd}}=5$ induced term. Such a nonrenormalizable second order contribution is deadly if there would be no possibility to extend the field content of the model in such a way that the interaction of $A_{\mu}$ with the new field leads to a compensating second order $d_{\text {sd }}=5$ contribution. The new field should have a lower spin (in order not to worsen the short distance situation) and the same Hermiticity property as $A_{\mu}$ i.e. it must be a $H$-field.

The compensation against another second order induced term from a first order $A A H$ interaction works perfect and converts the extended model into a renormalizable sl QFT ${ }^{10}$ $[17,18]$ (or [33] in the gauge theoretical setting in Krein space). It attributes a fundamental role to the $H$ coupling which is consistent with the principles of QFT. This compensating field is the Higgs field and the compensation is its raison d'être. Whereas in the case of SSB Nature has a choice between an inner symmetry and its breaking, she has no choice in the case of the Higgs model if she wants to be faithful to its causal localization principles.

This higher order compensation is a new phenomenon of $s \geq 1$ sl interactions which has no analog in $s<1 \mathrm{pl}$ interactions. Both the $L, Q_{\mu}$ pair condition as well as the higher order compensation mechanism are the prerequisites for the concepts of reactive and inert $s>1$ matter in the next section.

A full account of this preliminary sketch of low order sl perturbation theory is in preparation [35].

\section{Reactive and inert fields for $s \geq 1$}

As explained in the previous section the construction of positivity-obeying models of renormalizable QFT starts with an $L, Q_{\mu}$ pair in terms of a prescribed field content. $L^{\prime} s$ within the power-counting bound $d_{\text {sd }} \leq 4$ which couple $s \geq 1$ sl field among themselves or with $s<1$ pl fields exist for any spin, however, the pair condition is quite restrictive.

\footnotetext{
${ }^{10}$ A more detailed account will be presented in [35].
}

An example of a sl interaction $L$ which fails because it has no $Q_{\mu}$ partner is a self-interacting vector potential of the form $L=g\left(\partial_{\mu} A(x, e) \partial^{\mu} A(x, e)\right)^{2}$ with $d_{\text {sd }}(L)=4$. This is hardly surprising since this is also excluded in the gauge theoretic setting. In fact the requirement of invariance of the $S$-matrix under the BRST $\mathfrak{s}$ operation can also be implemented in the form of a pair condition $\mathfrak{s} L^{K}-\partial^{\mu} Q_{\mu}^{K}=0$ (where $K$ refers to a ghost-extended Krein space) [33].

It is interesting to note that in the 1970 s it was observed that the imposition of unitarity on the $S$-matrix in an indefinite metric pl setting of the Standard Model (i.e. using the Feynman propagator for the vector mesons) requires the presence of gauge symmetry and a $H$-field [36]. This was very close to the present view that the properties of self-interacting massive vector mesons are fully accounted for by positivity (which requires sl) and do not require the imposition of a Mexican hat potential for the purpose of mass-generation by allegedly spontaneously breaking gauge symmetry.

The use of the new positivity preserving SLF formalism resolves this somewhat paradoxical situation by showing that the field shifted Mexican hat potential is a second order induced $H$-self-interation in a $A-H$ model induced by positivity and the only causal localization which is consistent with it, namely sl. Given the field content of a massive vector meson and a Hermitian scalar field there is only one renormalizable model namely the abelian Higgs model.

The previous somewhat confused view resulted from the use of the positivity violating gauge setting (which only saves the perturbative unitarity of the $S$-matrix) together with the difficulty of distinguishing on- from off-shell properties by only looking at Feynman's graphical representation. That a more careful gauge theoretic treatment based on the EpsteinGlaser operator formulation of BRST gauge theory avoids confusions with SSB of gauge symmetry has been shown in Scharf's book [33].

The new sl setting shows that the "fattening" of massless helicity fields to their massive spin $s=|h|$ counterpart can be naturally achieved in the new positivity-maintaining sl setting [28,29]. That this is not possible in gauge theory may have been one of the reasons why the SSB idea entered the gauge theoretic presentation of the Higgs model.

Whereas the coupling of an abelian massive vector potential to a $H$ field (apart from the richer second order induction) takes place on the same conceptual level as that to a complex matter field ("massive" scalar QED) the situation changes radically in the presence of self-interacting vector mesons. As explained in the previous section one needs the presence of a $A-H$ coupling in order to compensate a second order induced $d_{\mathrm{sd}}=5$ self-interaction. This is also required in the gauge setting $[33,34]$.

The implementation of the positivity-maintaining sl perturbation theory is quite subtle. Improving the short distance behavior by converting $d_{\text {sd }}=s+1$ potentials into their 
$d_{s l}=1 \mathrm{sl}$ counterpart and the linearly related escort fields is only the first step. Already the next step which consist in constructing an sl interaction densities $L$ within the powercounting bound $d_{\text {sd }}(L) \leq 4$ in order to avoid a total delocalization already in second order is a major problem. It amounts to $L, Q_{\mu}$ pair condition whose heuristic content is the lowering of the $d_{\mathrm{sd}}\left(L^{P}\right)>4$ by splitting the pl interaction density into a $d_{s l} \leq 4 \mathrm{sl}$ part $L$ and a power-counting bound violating part which has the form of a divergence and hence can be disposed in the adiabatic limit.

As explained in the previous section this problem was solved for interactions which involve $s=1$ and lower spin fields with the important additional message that the second order consistency of self-interacting massive vector mesons requires the presence of the lower dimensional $H$. Presently one does not know to what extent this positivity preserving SLF renormalization approach leads to higher spin interactions. The following definition may be helpful in this pursuit.

Definition 1 A spin $s \geq 1$ field is called not reactive (or simply "inert") if it possesses no renormalizable sl interactions with $s^{\prime} \leq s$ fields in the sense of the new sl renormalization theory (sl pair condition and compensatory preservation of higher order renormalizability $\left.d_{\text {sd }} \leq 4\right)$. Inert sl matter exists only in the form of free fields (from which one can construct conserved currents, energy-momentum tensors and interactions with external fields).

The idea behind this definition is that the problems coming from the requirements of positivity preserving sl renormalizability increase with spin; a field which cannot interact with lower spin fields is a fortiori unable to preserve renormalizability in interactions with higher spin fields. Based on this picture one expects that higher spin fields above a certain still unknown critical spin will be inert. The old pl setting allowed only interactions between $s<1$ fields; the extension to $s=1$ required indefinite metric.

The infinite spin fields of WS are massless sl scalar fields [5] to which the higher spin sl tensor formalism is not directly applicable. The present picture is that WS is inert. But if against all expectations it turns out that reactive fields exist for all spins, this problem has to be re-investigated by studying the behavior of higher spin interactions in the Pauli-Lubanski limit.

Presently the $L, Q_{\mu}$ renormalization condition for simple sl interaction densities as e.g. $L \sim \gamma_{\mu \nu} A^{\mu} A^{\nu}$ with $\gamma_{\mu \nu}$ being the $s=2$ sl field are under investigation. Problems which involve self-interactions of graviton fields are more demanding.

The new sl renormalization theory is more fundamental than its old $\mathrm{pl}$ gauge theoretic counterpart since it reveals that renormalizability is nothing else than the implementation of positivity preserving causal localization realized in a perturbative setting. Since positivity is inseparably inter- laced with causal localization and a general (nonperturbative) theorem [20] revealed that interacting massive particles can be described in terms of sl fields (operators localized in arbitrary narrow space-like cones in the algebraic setting of the authors), the above definition of inert matter based on sl localization is well founded. In gauge theory such positivity preserving interpolating fields do not exist.

The sl renormalization theory can define inert fields but is not able to establish the mathematical existence of models associated to the renormalized perturbative series. Even if further calculations reveal (the rather improbable result) that no perturbative renormalizable $s>1$ sl reactive fields exist, the gain of profound understanding already obtained for $s=1$ interactions more than justifies the effort. In addition inert fields are not without interest since their lack of quantum field theoretical reactivity combined with their possible backreaction on classical gravitation (through the use of their energy momentum tensor in the form Einstein-Hilbert equation) make them potential candidates for dark matter.

A closely related problem in what way the use of sl fields could modify the old acausality properties observed in $s \geq 3 / 2$ fields coupled to external electromagnetic fields. It should be interesting to understand possible changes due to the use of sl fields.

The next section addresses problems of free massless potentials and their energy-momentum tensors. Closely related is the problem of how spin degrees of freedom pass smoothly to their massless helicity counterparts and its opposite i.e. how to disrobe the continuous passing from massless helicity degrees of freedom to massive spins ("fattening").

\section{The problem of the $s \geq 2 \mathrm{E}-\mathrm{M}$ tensor, coupling to gravity}

According to Noether's theorem continuous inner or spacetime symmetries in classical field theories lead to conservation laws for currents whose associated global charges are the infinitesimal generators of symmetries. These currents are constructed within the Lagrangian formalism by starting from the invariance of the classical action under these symmetry transformation. A particular useful object for the construction of the conserved currents associated to translation and Lorentz invariance is the divergence-free symmetric energy-momentum tensor $T_{\mu \nu}$ in terms of which these generators can be written as

$$
\begin{gathered}
P_{\mu}=\int T_{0 \mu} \mathrm{d}^{3} x, \quad M_{\mu \nu}=\int\left(x_{\mu} T_{0 \nu}-x_{\nu} T_{0 \mu}\right) \mathrm{d}^{3} x \\
T_{\mu \nu} \text { symmetric, }, \quad \partial^{\mu} T_{\mu \nu}=0
\end{gathered}
$$

Initial problems related to the lack of symmetry of the Noether formalism in the construction of the E-M tensor for 
fields with nontrivial spin/helicity were subsequently overcome by Belinfante's and Rosenfeld's construction of an "improved E-M tensor". In particular Rosenfeld's construction which avoids the usual Noether formalism in favor of a variational definition of the action with respect to a variable metric

$T_{\mu \nu}=\left.\frac{\delta}{\delta g_{\mu \nu}} \int \sqrt{-g} L \mathrm{~d}^{4} x\right|_{g_{\mu \nu}=\gamma_{\mu \nu}}$

led to a manifest symmetric Hilbert E-M tensor which plays an important role in the Einstein-Hilbert gravitation field equations of general relativity. Here the variational derivatives in $L$ include the covariant derivatives involving the Christoffel symbols; for its use in Minkowski space field theory the variable $g_{\mu \nu}$ is set equal to the Minkowski metric $\eta_{\mu \nu}$ after the variation has been carried out.

The remaining nontrivial problem (for which the classical Lagrange formalism for higher spin fields is of not much help) is the verification that the so constructed would be Poincare generators $P_{\mu}$ and $M_{\mu \nu}$ have the correct commutation relations with the higher spin $s \geq 1$ quantum fields. In the case of free fields this requires the use of their two-point functions which can be computed from the intertwiner functions associated to Wigner's unitary representation theory. Such a calculation avoids the (for higher spin) somewhat slippery parallelism of QFT with classical structures of Lagrangian quantization by direct use of the positivity-maintaining quantum causal localization principles.

For the verification of the correct commutation relations of the Poincaré symmetry generators (27) with the higher spin fields one needs the two-point functions of the fields which follow from their intertwiner functions. A recent explicit calculations of the higher spin intertwiners and their related two-point functions for integer spin fields can be found in [30].

Apparently the only such calculation in the older literature was made by Fierz [37]. He used the fact that integer spin fields are characterized by symmetric divergence- and traceless tensors obeying the Klein-Gordon equation and that the simplest conserved symmetric tensor of mass dimension ${ }^{11}$ four which is quadratic in these fields is of the form

$$
\begin{aligned}
& T_{\mu \nu}^{P, s}= F_{\mu \kappa, \times}^{P} F_{\nu}^{P, \kappa, \times}-\frac{1}{4} \delta_{\mu \nu} F_{\kappa \lambda, \times}^{P} F^{P, \kappa \lambda, \times} \\
&+g_{\mu \nu} \frac{m^{2}}{2} A_{\kappa, \times}^{P} A^{P, \kappa, \times}+m^{2} A_{\mu, \times}^{P} A_{\nu}^{P, \times} \\
& \text { with } F_{\mu \nu, \times}^{P}=\partial_{\mu} A_{\nu, \times}^{P}-(\mu \leftrightarrow \nu)
\end{aligned}
$$

Here $\times$ stands for the remaining spin $s$ tensor indices in $A^{P}$ or $F^{P}$. Actually the Fierz tensor is the one obtained

\footnotetext{
11 Fierz did not use Lagrangians but found his conserved tensor by first adding mass terms to the well-kown Maxwell E-M tensor and then extending the form of this conserved expression to higher spin.
}

from the variational definition (29) by omitting the $s>1$ contribution from the variation of the Christoffel symbols. ${ }^{12}$

By using the freedom to modify the tensor by terms of the form of spatial divergencies which do not contribute to the generators Fierz [37] was able to show that the $P_{\mu}$ permits the simpler representation

$$
P_{\mu}=\frac{(-1)^{s}}{4} \int \mathrm{d}^{3} x A_{\mu_{1} . . \mu_{s}}^{P} \overleftrightarrow{\partial}_{\mu} \overleftrightarrow{\partial}_{0} A^{P \mu_{1}--\mu_{s}}
$$

In this way he was able to derive the correct commutation relation of the Hamiltonian $P_{0}$ with the spin $s$ tensor fields. Using the higher spin two-point functions it is easy to show that the remaining $P$-components also satisfy the correct commutation relation with the higher spin fields.

Fierz probably believed that this will also be the case for the Lorentz generators but this turns out to be not correct. The variation (29) of the Christoffel symbol gives an additional contribution whose rewriting, using again the freedom to change densities without affecting the generators, leads to the correct commutators of $M_{\mu \nu}$ with the fields. The complete calculation, using the higher spin Jordan-Pauli commutator two-point function and a reduced form of the E-M tensor, are contained in $[28,29]$.

For these calculations it is important to accept the fact that the causal localization principle of QFT does not give preference to special $T_{\mu \nu}$; all causally localized covariant E-M tensors constructed in terms of positivity-obeying fields constructed from Wigner's unitary representation theory which lead to the correct Poincaré generators are valid. This is certainly not the case in a gauge theoretic Krein space setting where gauge variant operators are physically meaningless. The violation of the positivity of quantum probability also prevents to identify their formal localization properties with the physical causal localization.

This is of particular importance if one wants to overcome the aforementioned problems with massless tensors in the Weinberg-Witten theorem [22] which excludes the existence of pl E-M tensors. They can be traced back to the nonexistence of positivity-obeying massless $s \geq 1$ potentials. The impossibility of passing from massive spin to massless helicity in terms of $\mathrm{pl}$ higher spin fields is also the cause of the $s=2$ van Dam-Veltman-Zakharov discontinuity problem $[23,24]$; see also $[38,39]$.

The solution of these problems consists in expanding the pl Proca potentials into their massless sl counterparts and to notice that those contributions which contain $m^{-k}$ singularities are of the form of spatial divergencies which do not contribute to the total integral representing the $P_{\mu}$ and $M_{\mu \nu}$. Since their removal from the densities does not change the generators of the symmetries, one expects that the resulting expression decomposes into the $|h| \leq s$ helicity components

\footnotetext{
$\overline{12}$ I am indebted to K-H Rehren for this remark.
} 
and a massless $s=0$ contribution which together account for the original $2 s+1$ spin degrees of freedom.

The simplest exemplification of this construction is provided for the simpler model of a conserved vector current of a charge-carrying (complex) massive free spin $s$ Proca tensor

$j_{\mu}^{P}(x)=i A_{\kappa_{1} . . \kappa_{s}}^{P *}(x) \overleftrightarrow{\partial_{\mu}} A^{P, \kappa_{1} . . \kappa_{s}}(x)$

which already for $s=1$ fails to have a massless limit. Using the linear relation between the $s=1$ Proca potential, ${ }^{13}$ its sl counterpart and the escort field $\phi$ one finds for the corresponding sl current

$$
\begin{aligned}
& j_{\mu}(x, e)=i A_{\kappa}^{*}(x, e) \overleftrightarrow{\partial_{\mu}} A^{\kappa}(x, e) \\
& \quad=j_{\mu}^{P}(x)+\partial_{\kappa} G_{\mu}^{\kappa}(x, e)+m^{2} i \phi^{*} \overleftrightarrow{\partial_{\mu}} \phi
\end{aligned}
$$

with $\partial^{\kappa} G_{\kappa, \mu}=i A_{\kappa}^{P *} \overleftrightarrow{\partial_{\mu}} \partial^{\kappa} \phi+h . c .=i \partial^{\kappa}\left(A_{\kappa}^{P *} \overleftrightarrow{\partial_{\mu}} \phi\right)+$ h.c.

and since a spatial divergence does not contribute to the charge one obtains

$$
\begin{aligned}
\int j_{0}(x, e) \mathrm{d}^{3} x= & \int j_{0}^{P}(x) \mathrm{d}^{3} x \\
& +\int \partial^{0} G_{0,0}(x, e) \mathrm{d}^{3} x-m^{2} \int j_{0}^{\phi} \mathrm{d}^{3} x
\end{aligned}
$$

The last step consists in realizing that on can use $\partial \cdot A^{P}=0$ to convert also the mixed contribution $\partial^{0} G_{00}$ into a spatial divergence which carries an inverse power of the mass

$\partial^{0} G_{0,0}(x, e)=i \partial^{i} A_{0}^{P *} \overleftrightarrow{\partial_{i}} \phi+$ h.c. $=$ spatial divergence

$j_{0}^{P}(x)=j_{0}(x, e)+m^{2} j_{0}^{\phi}(x, e)+$ spatial divergence.

The massless limit of the first contribution is the $h=1$ component whereas the second term converges to the current $j_{\mu}^{\varphi}=\lim _{m \rightarrow 0} m^{2} j_{\mu}^{\phi}$ of a charge-carrying massless pl scalar field $\varphi(x)$ which accounts for the remaining $s_{3}=\emptyset$ degree of freedom.

The massless limit of the resulting sl current $j_{\mu}+m^{2} j_{\mu}^{\phi}$ is the sum of the helicity $h=1$ contribution accounting for two degrees of freedom, and a current of a scalar massless complex pl field $\varphi$ which carries the remaining one degree of freedom. For $s>1$ the decoupling is less simple. One needs additional transformations involving the sl field and its escorts (12) in order to decouple all helicity distributions and collect inverse mass singularities in spatial divergence contributions which do not contribute to the charge. In this way a suitably adapted escort formalism associates to any valid pl charge density a sl counterpart.

\footnotetext{
$\overline{13}$ In this case the commutation relation of the charge with $A_{\kappa}^{P}$ follows trivially.
}

Extending the decomposition of the pl current (36) into sl contributions to higher spins one finds again that the terms which contain inverse mass powers are of the form of spatial divergencies and hence do not contribute in the total charge. With a certain amount of work the remaining regular part of the sl density has a massless limit which consist of the completely decoupled helicity components contributing to the total charge

$Q=\bigoplus_{|h|=0}^{s} Q_{h}$,

which corresponds precisely to the smooth helicity composition of the field strength of the pl field (13) into its helicity components. The transformation of the pl charge density into its sl counterpart for $s>1$ is somewhat involved and the calculations can be found in [28,29].

The conversion of $\mathrm{pl}$ densities associated to $P_{\mu}$ and $M_{\mu \nu}$ into their sl counterparts and the disposal of terms with inverse mass powers and correspondingly higher short distance scale dimension ${ }^{14}$ in the infinite volume of the total charge proceeds the same way as for the current density. The resulting individual contributions to the massless limit correspond to helicity $|h| \leq s$ densities. In this way the WeinbergWitten no-go theorem can be sidestepped by the use of sl potentials.

The individual helicity contributions in the massless limit turn out to be sl E-M densities of the same algebraic form as their sl counterpart. One can also invert this relation by "fattening". For this purpose one only needs the highest helicity contribution and replace it by its fattened counterpart associated to its fattened two-point function which results by deforming the light-like $p^{\prime} s$ into their massive counterparts. For the presentation of all these results, we refer to [28, 29].

The DVZ discontinuity problem is solved by replacing the massive $s=2$ potential by that of its sl counterparts whose massless field strength is pure $h=2$. In this way the massive sl spin $s=2$ description moves smoothly towards its highest helicity component. The $\mathrm{pl}$ description is too rigid, it permits no massless limit.

For the WS matter there still exists no approximation in terms of known matter. The only idea which comes to one's mind is to try to approximate infinite spin matter in terms of the Pauli-Lubanski limit $s \rightarrow \infty$ at fixed P-L invariant $\kappa^{2}=m^{2} s(s+1)$. Although the sl fields of the WS matter are described in terms of scalar fields with transcendental sl intertwiners and two-point functions [5], it is not unreasonable to expect that a limiting E-M tensor constructed in this way has the correct commutation relations with the already

${ }^{14}$ All contributions carry the engineering dimension $d_{\text {eng }}=4$ of $T_{\mu \nu}$ and hence terms with inverse mass powers in front have $d_{\text {sd }}>4$ short distance dimension. 
known class of scalar WS fields [5] of which (15) is a special case.

It needs to be stressed that the use of massive sl fields in renormalized perturbation theory causes no problems with particle properties. Particles and their scattering amplitudes have lost the memory of the sl localization of the interpolating quantum fields whose main role is to carry the causal localization principles of QFT which are not directly implementable in terms of particles.

In the absence of interactions the clash between tightness of localization and positivity is limited to massless $s \geq 1$ tensor potentials and their spinorial counterparts, but in the presence of interaction this clash causes the breakdown of renormalizability for all positivity preserving interactions whose first order interaction densities contain at least one $s \geq 1 \mathrm{pl}$ localized free field. Behind the breakdown of pl renormalizability in the presence of interactions and the nonexistence of higher helicity E-M tensors (the Weinberg-Witten phenomenon) hides the same clash between positivity and pl localization; hence it is not surprising that the shared remedy is the use of sl instead of $\mathrm{pl}$ potentials.

The resulting directional dependence of sl fields does not affect local observables and the $S$-matrix and, unlike gauge theory, prevents the important particle interpolating fields from becoming unphysical. The use of sl fields shifts the frontier of renormalization theory by turning a $s \geq 1$ interacting nonrenormalizable field content into an (under certain conditions) renormalizable interaction in such a way that the heuristic content of the pl Lagrangian setting is maintained. The mechanism is a kind of "peeling off" renormalizability violating leading short distance contributions in the form of spacetime divergences $\partial^{\mu} Q_{\mu}$ in the adiabatic limit. This corresponds to the disposal of high $d_{\text {sd }}$ contributions in the total charge in passing from pl to sl charge densities.

Based on an appropriate formulation of Gauss' law in the setting of local quantum physics it was possible without using perturbation theory to show that the localization of electric charge carriers cannot be tighter than space-like half-lines (arbitrary narrow space-like cones in the algebraic setting) [40] and that their sl "stiffness" in the massless case causes the spontaneous breaking of Lorentz invariance in chargecarrying sectors of QED [41]. But registrations of charged particles in counters remain sharply localized events and the noncompact charge localization manifests itself indirectly in the larger spacetime region in which counters register the presence of charges.

The situation changes if massless sl E-M tensor densities are used in the Einstein-Hilbert equation to calculate gravitational backreactions [42] of massless higher helicity quantum matter. In this case one uses the sl E-M density of the Hilbert action (29) and the Einstein-Hilbert field equa- tions which couple this density to the gravitational field and one expects that the "stringiness" of the E-M tensor leaves its imprint on the gravitational backreaction.

This is particularly interesting in the case of the E-M tensor associated to the massless WS matter which only exists in the sl localized form. In this case one would expect that the backreaction leads to a kind of gravitational "cosmic strings". Since WS matter is expected to be inert with respect to interactions ordinary with quantum fields (see previous section) such non-gravitational inertness is suggestive of of dark matter. However, there are additional observational requirements from astrophysics (e.g. the "hovering" of dark matter around galaxies) which are difficult to reconcile with the masslessness of WS.

The absence of reactivity may not be limited to infinite spin. Disposing contributions of high short distance dimensions in the adiabatic limit by using sl localization in interactions of spin $s$ fields with lower spin fields (including self-interactions) is expected to become increasingly difficult with increasing spin. In this case there could exist a maximal spin above which matter becomes inert in the sense of Sect. 4 except for causing gravitational backreactions. Massive inert matter can more easily be reconciled with astrophysical restrictions. Presently we only know that $s=1$ is still in the reactive region; in the self-interacting case the model is saved by the compensating presence of the Higgs field.

The SLF extension to higher spin interactions is still very much in its infancy. Without additional nontrivial calculations it is not possible to say which pl couplings will fail to admit $L, Q_{\mu}$ pairs or lead to higher order induced powercounting bound violating terms which cannot be compensated for by adding interactions with lower spin fields.

\section{Concluding remarks}

The possible existence of forms of inert matter enriches the discussion as regards dark matter in an interesting way. Inert WS matter seems to run into problems with astrophysical data which show that dark matter hovers around galaxies in the form of a halo which probably excludes "fleeting" massless matter, though noncompact non-interacting massless matter may behave quite different from photons. Inert massive $s>1$ matter (whose existence is still an unresolved theoretical problem, see Sect. 4), can presently not be excluded by astrophysical observations.

There is presently no serious problem with more conventional proposals which identify dark matter with matter of low reactivity in the form of cold dark matter. Such proposals become however increasingly problematic if refined earthly detection experiments limit the reactivity of the astrophysical dark matter in a way which cannot be reconciled with 
properties of known particles. A continuing failure of registering dark matter through its interactions with ordinary matter makes it increasingly difficult to distinguish darkness from inertness (=total darkness, apart from backreaction on gravitation).

With refinements of astrophysical observations on the one hand and the failure to see the direct effects of dark matter in particle counters one may eventually enter a future "catch 22 situation" in the sense that completely inert dark matter is on the one hand consistent with its apparently exclusive gravitational manifestations, but may get into conflict with the role which cosmologists attribute to it in the Big Bang formation of ordinary matter.

Any particle counter observation of a new form of matter for which there are good reasons to understand it as a manifestation of the ubiquitous galactic dark matter will eliminate inert matter from the list of dark matter candidates. But this would not diminish the important theoretical role of WS as a catalyzer of new ideas concerning the interplay between Hilbert space positivity, localization and short distance behavior for interactions involving higher spin, $s \geq 1$, fields.

Independent of the problem of inert matter and its possible relation with dark matter, Wigner's 1939 discovery of the WS representation class of the Poincare group and the recent success in the understanding of its causal localization properties constitute important achievements which are presently unraveling unknown regions of QFT which remains our experimentally most successful and theoretically most foundational theory of Nature's physical properties.

Acknowledgements I thank Jens Mund and Jakob Yngvason for their continued interest in the issue of sl fields associated to the third Wigner class whose construction was the result of a past joint collaboration [5]. I am indebted to Christian Köhler for sending me a copy of his thesis before publication and I thank José Gracia-Bondia for a critical reading of the manuscript. Last not least I am indebted to Henning Rehren for sharing with me his interesting observation about how the string-local energy-momentum tensor, which is for spin $s \geq 1$ more natural, preserves the $2 s+1$ degrees of freedom in the massless helicity limit.

Open Access This article is distributed under the terms of the Creative Commons Attribution 4.0 International License (http://creativecomm ons.org/licenses/by/4.0/), which permits unrestricted use, distribution, and reproduction in any medium, provided you give appropriate credit to the original author(s) and the source, provide a link to the Creative Commons license, and indicate if changes were made. Funded by SCOAP ${ }^{3}$.

\section{References}

1. E.P. Wigner, On unitary representations of the inhomogeneous Lorentz group. Ann. Math. 40, 149 (1939)

2. S. Weinberg, The Quantum Theory of Fields I (Cambridge University Press, Cambridge, 1991)
3. H. Epstein, V. Glaser, Ann. Inst. Poincaré A 19, 211 (1973)

4. R. Longo, V. Morinelli, K.H. Rehren, Where infinite spin particles are localizable. Commun. Math. Phys. 345, 587 (2016)

5. J. Mund, B. Schroer, J. Yngvason, String-localized quantum fields and modular localization. Commun. Math. Phys. 268, 621 (2006)

6. L.F. Abbott, Massless particles with continuous spin indices. Phys. Rev. D 13(8), 2291 (1976)

7. P. Schuster, N. Toro, A gauge field theory of continuous-spin particles. JHEP 20, 10 (2013)

8. J. Yngvason, Zero-mass infinite spin representations of the Poincaré group and quantum field theory. Commun. Math. Phys. 18, 195 (1970)

9. R. Brunetti, D. Guido, R. Longo, Modular localization and Wigner particles. Rev. Math. Phys. 14, 759 (2002)

10. J. Mund, String-localized quantum fields, modular localization, and gauge theories, in New Trends in Mathematical Physics, Selected Contributions of the XVth International Congress on Mathematical Physics, ed. by V. Sidoravicius (Springer, Dordrecht, 2009), p. 495

11. C. Köhler, On localization properties of quantum fields with zero mass and infinite spin. Thesis, University of Vienna (2015)

12. T.P. Hack, On the backreaction of scalar and spinor quantum fields. Ph.D. thesis. arXiv:1008.1776

13. R. Haag, Local Quantum Physics (Springer, Heidelberg, 1996)

14. B. Schroer, Modular localization and the $d=1+1$ formfactor program. Ann. Phys. 275, 190 (1999)

15. G. Lechner, Algebraic constructive quantum field theory: integrable models and deformation techniques. arXiv:1503.0322

16. S. Allazzawi, G. Lechner, Inverse scattering and locality in integrable quantum field theories. arXiv:1608.02359

17. B. Schroer, Peculiarities of massive vectormesons. Eur. Phys. J. C 75, $365(2015)$

18. B. Schroer, Beyond gauge theory: Hilbert space positivity and its connection with causal localization in the presence of vector mesons. Eur. Phys. J. C. 76, 378 (2016)

19. B. Schroer, Is inert matter from indecomposable positive energy "infinite spin" representations the much sought-after dark matter? arXiv:0802.2098v3 (unpublished)

20. D. Buchholz, K. Fredenhagen, Locality and the structure of particle states. Commun. Math. Phys. 84, 1 (1982)

21. M.A. Vassiliev, Higher spin superalgebras in any dimension and their representations. J. High Energy Phys. 12, 046 (2004)

22. S. Weinberg, E. Witten, Limits on massless particles. Phys. Lett. B 96, 59 (1980)

23. H. van Dam, M. Veltman, Massive and massless Yang-Mills and gravitational fields. Nucl. Phys. B 22, 397 (1970)

24. V.I. Zakharov, Linearized gravitation theory and the graviton mass. JETP Lett. 12, 312 (1970)

25. M. Plaschke, J. Ingvason, Massless string-localized quantum fields for any helicity. J. Math. Phys. 53, 04230 (2012)

26. R.S. Streater, A.S. Wightman, PCT Spin and Statistics and All That (Benjamin, New York, 1964)

27. H. Ruegg, M. Ruiz-Altaba, The Stueckelberg field. Int. J. Mod. Phys A19, 3265 (2003)

28. J. Mund, K.-H. Rehren, B. Schroer, Relations between positivity, localization and degrees of freedom: the WeinbergWitten theorem and the van Dam-Veltman-Zakharov discontinuity. arXiv: 1705.04408

29. J. Mund, K.-H. Rehren, B. Schroer, Helicity decoupling in the massless limit of massive tensor fields. arXiv:1705.04407

30. J. Mund, E.T. de Oliveira, String-local free vector and tensor potentials for massive particles with any spin: 1 bosons. Commun. Math. Phys. arXiv:1609.01667 (to appear)

31. J. Fröhlich, G. Morchio, F. Strocchi, Infrared problem and spontaneous breaking of the Lorentz group in QED. Phys. Lett. B 89, 61 (1979)

32. D. Yenni, S. Frautschi, H. Suura, Ann. Phys. 13, 370 (1961) 
33. G. Scharf, Quantum Gauge Theory, A True Ghost Story (Wiley, New York, 2001)

34. M. Duetsch, J.M. Gracia-Bondia, F. Scheck, J. Varilly, Quantum gauge models without classical Higgs mechanism. Eur. Phys. J. C 80, 599 (2012)

35. J. Mund, B. Schroer, How the Higgs potential got its shape (forthcoming)

36. J.M. Cornwall, D.N. Levin, G. Tiktopoulos, Derivation of gauge invariance from high-energy unitarity bounds on the S-matrix. Phys. Rev. D 10, 1145 (1974)

37. M. Fierz, Über die relativistische Theorie kräftefreier Teilchen mit beliebigem Spin. Helv. Phys. Acta 12, 3 (1939)

38. M. Porrati, Universal limits on massless high-spin particles. Phys. Rev. D 78, 06506 (2008)
39. X. Bekaert, N. Boulanger, P. Sundell, How higher-spin gravity passes the spin 2 barrier: no go theorems versus yes-go examples. Rev. Mod. Phys. 84, 987 (2012). arXiv: 1007.0435

40. D. Buchholz, The physical state space of quantum electrodynamics. Commun. Math. Phys. 85, 49 (1982)

41. J. Fröhlich, The charged sectors of quantum electrodynamics in a framework of local observables. Commun. Math. Phys. 66, 223 (1979)

42. D. Dappiagi, K. Fredenhagen, N. Pinamonti, Stable cosmological models driven by a free quantum scalar field. Phys. Rev. D 77, 194915 (2008) 